\title{
Radiative cooling of solar cells
}

\author{
LinXiao Zhu, ${ }^{1}$ Aaswath Raman, ${ }^{2}$ Ken Xingze Wang, ${ }^{1}$ \\ Marc Abou Anoma, ${ }^{3}$ and Shanhul Fan ${ }^{2, *}$ \\ ${ }^{1}$ Department of Applied Physics, Stanford University, Stanford, California 94305, USA \\ ${ }^{2}$ Department of Electrical Engineering, Ginzton Laboratory, Stanford University, Stanford, California 94305, USA \\ ${ }^{3}$ Department of Mechanical Engineering, Stanford University, Stanford, California 94305, USA \\ ${ }^{*}$ Corresponding author: shanhui@stanford.edu
}

Received 31 March 2014; revised 13 May 2014; accepted 17 May 2014 (Doc. ID 209022); published 22 July 2014

\begin{abstract}
Standard solar cells heat up under sunlight. The resulting increased temperature of the solar cell has adverse consequences on both its efficiency and its reliability. We introduce a general approach to radiatively lower the operating temperature of a solar cell through sky access, while maintaining its solar absorption. We first present an ideal scheme for the radiative cooling of solar cells. For an example case of a bare crystalline silicon solar cell, we show that the ideal scheme can passively lower its operating temperature by $18.3 \mathrm{~K}$. We then demonstrate a microphotonic design based on real material properties that approaches the performance of the ideal scheme. We also show that the radiative cooling effect is substantial, even in the presence of significant convection and conduction and parasitic solar absorption in the cooling layer, provided that we design the cooling layer to be sufficiently thin. () 2014 Optical Society of America
\end{abstract}

OCIS codes: (350.6050) Solar energy; (230.5298) Photonic crystals; (290.6815) Thermal emission.

http://dx.doi.org/10.1364/OPTICA.1.000032

From Shockley and Queisser's analysis, a single junction solar cell has a theoretical upper limit for power conversion efficiency of around $33.7 \%$ [1] under the AM1.5 solar spectrum. Thus, while a solar cell absorbs most incident solar irradiance $[2,3]$, there is intrinsically a significant portion of absorbed solar irradiance that is not converted into electricity, and instead generates heat that, in turn, heats up the solar cell. In practice, the operating temperature of a solar cell in outdoor conditions is typically $50^{\circ} \mathrm{C}-55^{\circ} \mathrm{C}$ or higher [4-6]. This heating has significant adverse consequences for the performance and reliability of solar cells. The conversion efficiency of solar cells typically deteriorates at elevated temperatures. For crystalline silicon solar cells, every temperature rise of $1 \mathrm{~K}$ leads to a relative efficiency decline of about $0.45 \%$ [7]. Furthermore, the aging rate of a solar cell array doubles for every $10 \mathrm{~K}$ increase in its operating temperature [8]. Therefore, there is a critical need to develop effective strategies for solar cell cooling. Current approaches include conduction of heat to dissipation surfaces [9], forced air flow [10], hot water generation in combined photovoltaic/thermal systems [11], and heat-pipe-based systems [1ㅡ, $\underline{13}]$.
In this paper, we propose the use of radiative cooling to passively lower the temperature of solar cells operating under direct sunlight. The basic idea is to place a thin material layer that is transparent over solar wavelengths but strongly emissive over thermal wavelengths on top of the solar cell. Such a layer does not degrade the optical performance of the solar cell, but does generate significant thermal radiation that results in solar cell cooling by radiatively emitting heat to outer space.

The Earth's atmosphere has a transparency window between 8 and $13 \mu \mathrm{m}$, which coincides with the wavelength range of thermal radiation from terrestrial bodies at typical temperatures. Terrestrial bodies can therefore cool down by sending thermal radiation into outer space. Both nighttime $[\underline{14}-\underline{23}]$ and daytime $[\underline{15}, \underline{24}-27]$ radiative cooling has been studied previously. Most of these studies sought to achieve an equilibrium temperature that is below the ambient air temperature. In daytime, achieving radiative cooling below ambient temperature requires reflecting over $88 \%$ of incident solar irradiance [24]. Solar cells, on the other hand, must absorb sunlight. Thus, unlike most previous radiative cooling works $[\underline{15}, \underline{24}-\underline{26}]$, we do not seek to achieve an equilibrium 
temperature that is below the ambient. Instead, for a solar cell with a given amount of heat generated by solar absorption, our objective is to lower its operating temperature as much as possible, while maintaining its solar absorptance.

Without loss of generality, we consider crystalline silicon solar cells, representing about 90\% [28] of solar cells produced worldwide in 2008. Crystalline silicon can absorb a considerable amount of solar irradiance, and has a very small extinction coefficient at thermal wavelengths at typical terrestrial temperatures. Thus, crystalline silicon solar cells represent a worstcase scenario as far as radiative cooling is concerned since they emit very small amounts of thermal radiation. In our simulations, as a model of the optical and thermal radiation properties of a silicon solar cell, we consider the structure shown in Fig. 1(a), which consists of a $200-\mu \mathrm{m}$-thick crystalline Si layer [29] on top of an aluminum (Al) backreflector. The silicon is p-doped with a concentration of $1.5 \times 10^{16} \mathrm{~cm}^{-3}$, which represents the typical base material of a crystalline silicon solar cell [29]. The dielectric constant of the doped silicon for optical simulation is obtained from [30]. To achieve radiative cooling of the cell, we then add a variety of structures on top of the solar cell and facing the sky, as shown in Figs. $1(\mathrm{~b})-1(\mathrm{~d})$. These additional structures are typically made of silica.

To analyze the cooling properties of each of the structures shown in Fig. 1, we use the following procedure, which integrates electromagnetic and thermal simulations. We start with an electromagnetic (EM) simulation of the structure using the rigorous coupled wave analysis (RCWA) method [31]. At thermal wavelengths, the simulation results in an absorptivity/ emissivity spectrum $\epsilon(\lambda, \Omega)$. This spectrum is then used to compute the cooling power:

$$
P_{\text {cooling }}\left(T_{\text {Emit }}\right)=P_{\text {rad }}\left(T_{\text {Emit }}\right)-P_{\text {atm }}\left(T_{\text {amb }}\right),
$$

where

$$
P_{\text {rad }}\left(T_{\text {Emit }}\right)=\int \mathrm{d} \Omega \cos \theta \int_{0}^{\infty} \mathrm{d} \lambda I_{B B}\left(T_{\text {Emit }}, \lambda\right) \epsilon(\lambda, \Omega)
$$

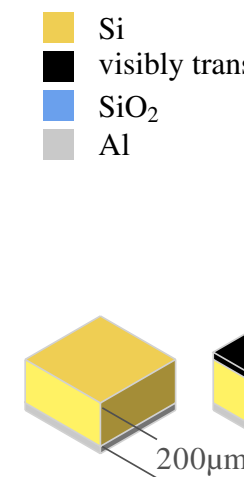

(a)

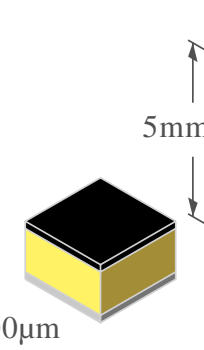

(b)

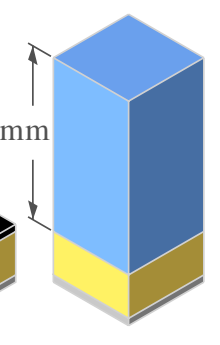

(c)

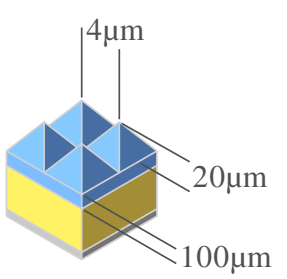

(d)
Fig. 1. $3 \mathrm{D}$ crystalline silicon solar cell structures. (a) Bare solar cell with $200-\mu \mathrm{m}$-thick uniform silicon layer, on top of an Al backreflector. (b) Thin visibly transparent ideal thermal emitter on top of the bare solar cell. (c) 5-mm-thick uniform silica layer on top of the bare solar cell. (d) $2 \mathrm{D}$ square lattice of silica pyramids and a $100-\mu \mathrm{m}$-thick uniform silica layer, on top of the bare solar cell. is the power radiated by the structure per unit area. Here $T_{\text {Emit }}$ is taken to be the temperature of the top surface and will be determined self-consistently when we combine the EM and thermal simulations. $\int \mathrm{d} \Omega$ is the solid angle integral over a hemisphere. $I_{B B}(T, \lambda)$ is the spectral radiance of a blackbody at temperature $T$, and

$$
P_{\mathrm{atm}}\left(T_{\mathrm{atm}}\right)=\int \mathrm{d} \Omega \cos \theta \int_{0}^{\infty} \mathrm{d} \lambda I_{B B}\left(T_{\mathrm{amb}}, \lambda\right) \epsilon(\lambda, \Omega) \epsilon_{\mathrm{atm}}(\lambda, \Omega)
$$

is the power absorbed from the ambient atmosphere. The angle-dependent emissivity of the atmosphere is given by [19] as $\epsilon_{\text {atm }}(\lambda, \Omega)=1-t(\lambda)^{1 / \cos \theta}$, where $t(\lambda)$ is the atmospheric transmittance in the zenith direction $[32,33]$. To evaluate the cooling power, we calculate the emissivity/absorptivity with a spectral resolution of $2 \mathrm{~nm}$ from 3 to $30 \mu \mathrm{m}$, and with $5 \mathrm{deg}$ angular resolution across the hemisphere. With these spectral and angular resolutions, the cooling power has converged within $0.5 \%$ relative accuracy. We note that we take into account the temperature dependence of the permittivity of doped silicon [30] in the electromagnetic simulations, and the absorptivity/emissivity spectra are calculated for various temperatures of solar cells. The permittivity of silica has negligible temperature dependence.

We also use the electromagnetic simulation to determine the solar absorption profile within the silicon solar cell structure. By assuming a total heating power of the solar cell, which in practice corresponds to the difference between the absorbed solar power and extracted electrical power, we can then determine a spatially dependent heat generation rate $\dot{q}(z)$ within the silicon solar cell region.

The results from the electromagnetic simulations are then fed into a finite-difference-based thermal simulator, where we simulate the temperature distribution within the structure by solving the steady-state heat diffusion equation:

$$
\frac{d}{d z}\left[\kappa(z) \frac{d T(z)}{d z}\right]+\dot{q}(z)=0
$$

where $T(z)$ is the temperature distribution. In this equation, the thermal conductivity $\kappa$ of silicon and of silica are taken to be $148 \mathrm{~W} / \mathrm{m} / \mathrm{K}$ and $1.4 \mathrm{~W} / \mathrm{m} / \mathrm{K}$, respectively [34]. The schematic of the simulation is shown in Fig. 2 , where the vertical direction aligns with the $z$ axis.

The simulated region consists of the silicon solar cell and the silica structure on top of it. At the upper surface, we assume, as a boundary condition,

$$
-\left.\kappa(z) \frac{d T(z)}{d z}\right|_{\text {top }}=P_{\text {cooling }}\left(T_{\text {Emit }}\right)+h_{1}\left(T_{\text {Emit }}-T_{\text {amb }}\right)
$$

to take into account both the cooling effect due to radiation, as well as additional nonradiative heat dissipation due to convection and conduction, as characterized by $h_{1}$. At the lower surface, we assume a boundary condition 


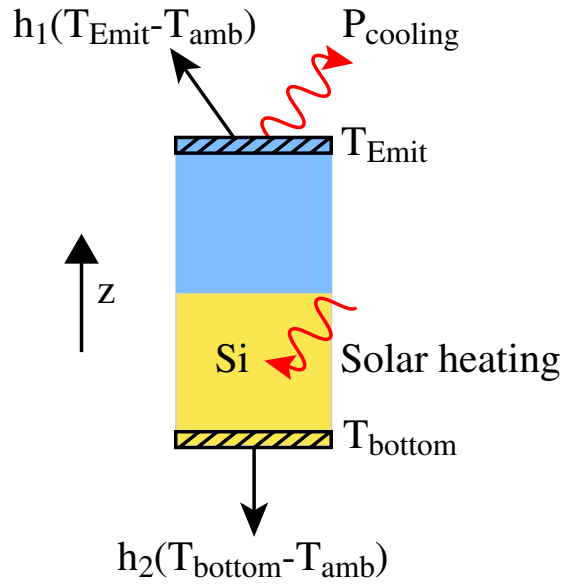

Fig. 2. Schematic of thermal simulation. $h_{1}$ and $h_{2}$ are the nonradiative heat exchange coefficients at the upper and lower surfaces, respectively. Ambient temperature is $T_{\mathrm{amb}}$.

$$
\left.\kappa \frac{d T(z)}{d z}\right|_{\text {bottom }}=h_{2}\left(T_{\text {bottom }}-T_{\text {amb }}\right)
$$

to characterize the nonradiative heat loss of the lower surface. The solution of the heat equation results in a temperature distribution $T$. The temperature of the upper surface is then used as $T_{\text {Emit }}$ in Eq. () and input back into the boundary condition; the heat equation is then solved again. This process is iterated until self-consistency is reached, i.e., until the temperature of the upper surface no longer changes with iteration. The operating temperature of the solar cell is then defined as the spatially averaged temperature inside the silicon region.

We use a $1 \mathrm{D}$ thermal model for such a $3 \mathrm{D}$ structure because the temperature variation in the horizontal direction is sufficiently small. As a simple estimation, consider the temperature difference $\Delta T$ between the center of the pyramid and the edge for the structure in Fig. 1(d). Such a temperature difference results in a power flow of $\kappa \Delta T / d$, where $d \approx 2 \mu \mathrm{m}$ is the distance between the center and the edge. Such a power flow should be less than the cooling power of the device, which is $208 \mathrm{~W} / \mathrm{m}^{2}$ at $T=300 \mathrm{~K}$, and $554 \mathrm{~W} / \mathrm{m}^{2}$ at $T=350 \mathrm{~K}$, with $T_{\mathrm{amb}}=300 \mathrm{~K}$. Thus, we estimate $\Delta T \approx 8 \times 10^{-4} \mathrm{~K}$. This is sufficiently small to justify the use of a $1 \mathrm{D}$ thermal model.

As a typical scenario, we consider the ambient on both sides of solar cell to be at $300 \mathrm{~K}$. The nonradiative heat exchange coefficients are $h_{1}=12 \mathrm{~W} / \mathrm{m}^{2} / \mathrm{K}$ and $h_{2}=6 \mathrm{~W} / \mathrm{m}^{2} / \mathrm{K}$, corresponding to wind speeds of $3 \mathrm{~m} / \mathrm{s}$ and $1 \mathrm{~m} / \mathrm{s}$ [25]. The annual average wind speed at a height of $30 \mathrm{~m}$ in most parts of the United States is at or below $4 \mathrm{~m} / \mathrm{s}$ [35]. The wind speed at a height of $10 \mathrm{~m}$, which is more relevant to solar cell installations, can be estimated from the horizontal wind speed at $30 \mathrm{~m}$ by using the $1 / 7$ power law [36], to be below $4 \times(10 / 30)^{1 / 7}=3.4 \mathrm{~m} / \mathrm{s}$. $h_{2}$ is chosen to reflect the fact that the wind speed on the unexposed rear side of solar cells is smaller than the exposed front side [5].

Using the numerical procedure outlined above, we now present simulation results on the configurations shown in Fig. 1. Without any radiative cooling structure on top, the solar cell structure shown in Fig. 1(a) (which we will refer to as the "bare solar cell" below) heats up substantially above the ambient for various solar heating powers (Fig. $\underline{3}$, blue curve). At $800 \mathrm{~W} / \mathrm{m}^{2}$ solar heating power, corresponding approximately to the expected heat output of a crystalline solar cell under peak unconcentrated solar irradiance, the bare solar cell operates at $42.3 \mathrm{~K}$ above ambient.

To radiatively cool the solar cell, our design principle is to place on top of the bare solar cell a layer that emits strongly in the thermal wavelength range, while being transparent at solar wavelengths. To illustrate the theoretical potential of this idea, we first consider the ideal scenario [Fig. 1(b)] where the added layer has unity emissivity in the wavelength range above $4 \mu \mathrm{m}$, and has zero emissivity below $4 \mu \mathrm{m}$. Such a layer has maximal thermal radiative power, and, in the meantime, does not absorb sunlight; hence, it has maximal cooling power. With such an ideal layer added, the solar cell operates at a substantially lower temperature (Fig. $\underline{3}$, green curve), as compared to the bare solar cell case. At $800 \mathrm{~W} / \mathrm{m}^{2}$ solar heating power, the solar cell with the ideal cooling layer operates at a temperature that is $18.3 \mathrm{~K}$ lower as compared to the bare solar cell. The calculation here points to the significant theoretical potential of using radiative cooling in solar cells.

To implement the concept of radiative cooling for solar cells we consider the use of silica as the material for the cooling layer. Pure silica is transparent over solar wavelengths and has pronounced phonon-polariton resonances, and hence emissivity, at thermal wavelengths. Standard solar panels are typically covered with glass, which contains $70 \%$ to $80 \%$ silica [37] and, therefore, potentially provide some radiative cooling benefit already. As we will show here, however, the cooling performance of a thick and flat layer of silica is significantly lower than the theoretical potential. Moreover, as we discuss later, typical solar absorption in glass significantly counteracts the potential radiative benefit it provides. Emulating the geometry of a typical solar panel cover glass, we examine a thermal emitter design consisting of a 5-mm-thick uniform pure silica

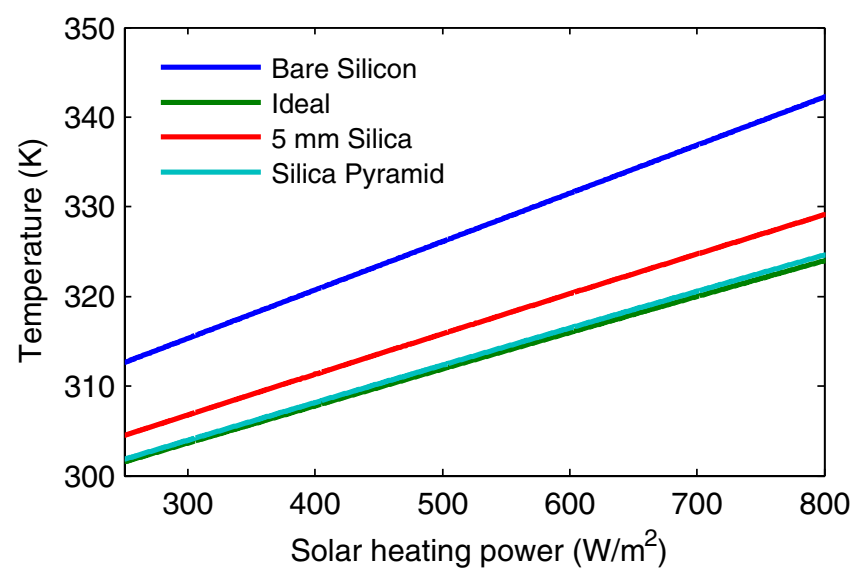

Fig. 3. Operating temperature of solar cells with the thermal emitter designs in Fig. 1, for different solar heating power. The nonradiative heat exchange coefficients are $h_{1}=12 \mathrm{~W} / \mathrm{m}^{2} / \mathrm{K}$ (corresponding to $3 \mathrm{~m} / \mathrm{s}$ ), and $h_{2}=6 \mathrm{~W} / \mathrm{m}^{2} / \mathrm{K}$ (corresponding to $1 \mathrm{~m} / \mathrm{s}$ ). The ambient temperatures at the top and the bottom are both $300 \mathrm{~K}$. 
layer on top of the bare solar cell [Fig. 1(c)]. The use of the 5 -mm-thick uniform silica layer (Fig. $\underline{3}$, red curve) does enable an operating temperature considerably lower than that of the bare solar cell. However, the radiative cooling performance of 5 -mm-thick uniform silica is inferior to the ideal case. At $800 \mathrm{~W} / \mathrm{m}^{2}$ solar heating power, solar cell with 5 -mm-thick uniform silica operates at a temperature $5.2 \mathrm{~K}$ higher than the ideal case (Fig. $\underline{3}$, green curve).

We now present a microphotonic design, shown in Fig. 1(d), that has performance that approaches the ideal case. The thermal emitter design consists of a 2D square lattice of silica pyramids, with $4 \mu \mathrm{m}$ periodicity and $20 \mu \mathrm{m}$ height, on top of a $100-\mu \mathrm{m}$-thick uniform silica layer. We refer to this design as a "silica pyramid" design. Such a silica pyramid design substantially lowers the temperature of the solar cell (Fig. $\underline{3}$, cyan curve). It considerably outperforms the 5-mmthick uniform silica design, and has performance nearly identical to the ideal scheme. At $800 \mathrm{~W} / \mathrm{m}^{2}$ solar heating power, the temperature reduction of the silica pyramid design is 17.6 K, compared with the bare solar cell. Using [7], we estimate that such a temperature drop should result in a relative efficiency increase of about $7.9 \%$. If the solar cell efficiency is $20 \%$, this temperature drop corresponds to a $1.6 \%$ absolute efficiency increase, which is a significant improvement of solar cell efficiency.

To reveal the mechanism underlying the different cooling performance, we examine the emissivity spectra of the different designs at thermal wavelengths in Fig. $\underline{4}$. The bare solar cell has only small emissivity at thermal wavelengths (Fig. $\underline{4}$, blue curve). Accordingly, the solar cell heats up substantially.

For the ideal case (Fig. $\underline{4}$, green curve), the emissivity at thermal wavelengths is unity, which enables the structure to radiatively cool maximally.

For the uniform silica layer (Fig. $\underline{4}$, red curve), the emissivity at thermal wavelengths is considerable. However, the emissivity spectrum shows two large dips near 10 and $20 \mu \mathrm{m}$. These dips correspond to the phonon-polariton resonances of silica. At these wavelengths, silica has a large extinction coefficient, and there is a strong impedance mismatch between silica and

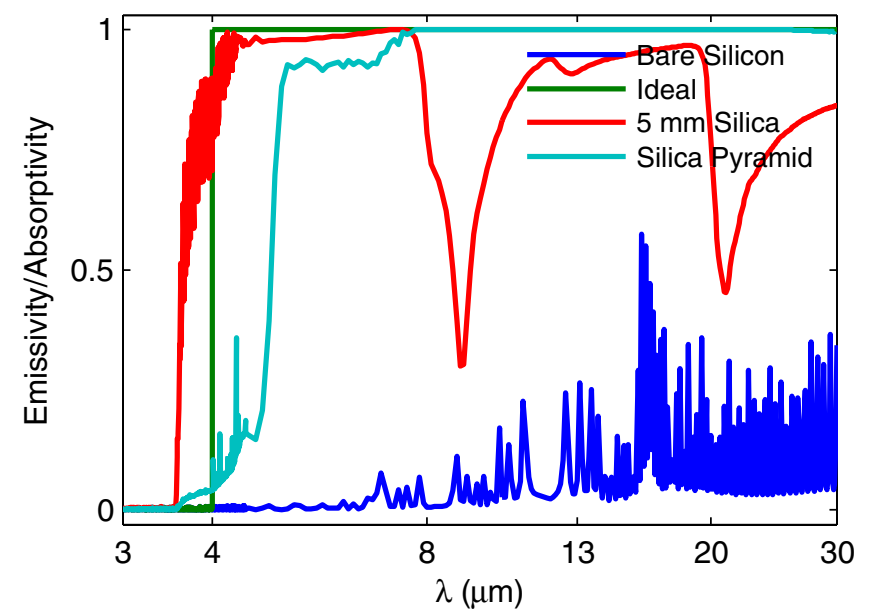

Fig. 4. Emissivity and absorptivity spectra of solar cells with different thermal emitter designs in Fig. 1 , for normal direction and after averaging over polarizations. The temperature of solar cells is $300 \mathrm{~K}$. air. The large impedance mismatch results in large reflectivity, and accordingly small absorptivity/emissivity. These dips coincide with the $8-13 \mu \mathrm{m}$ atmospheric transmission window and a secondary atmospheric transmission window at 20-25 $\mu \mathrm{m}$ [26], respectively. Moreover, the dip near $10 \mu \mathrm{m}$ coincides with the peak blackbody radiation wavelength of $9.7 \mu \mathrm{m}$ for the typical terrestrial temperature of $300 \mathrm{~K}$. Therefore, the cooling capability of 5-mm-thick uniform silica is inferior to the ideal case.

The silica pyramid design, however, has emissivity very close to unity at the whole range of thermal wavelengths (Fig. $\underline{4}$, cyan curve). Comparing with the uniform silica structure, we observe that the use of the pyramid eliminates the two dips near 10 and $20 \mu \mathrm{m}$. In the silica pyramid design, the absence of sharp resonant features associated with silica phononpolariton resonances and, hence, broadband near-unity absorption is achieved because the pyramids provide a gradual refractive index change to overcome the impedance mismatch between silica and air at a broad range of wavelengths, including the phonon-polariton resonant wavelengths.

We have focused on designing a thin material layer that generates significant thermal radiation, while being optically transparent so that it does not degrade the optical performance of the solar cell. The silica pyramid has a size of several micrometers, and is significantly larger than wavelengths in the solar spectrum. Due to this strong size contrast, the silica pyramid does not degrade solar absorptivity. This remains true even in the presence of an antireflection layer. As an example, we show that, for a solar cell with a $75 \mathrm{~nm}$ silicon nitride layer on top as antireflection coating, the silica pyramid design does not degrade the solar absorptivity (see Supplement 1). Our proposed silica pyramid structure for enhancing thermal radiation is thus compatible with antireflection coating design, by not degrading the solar absorptivity of the solar cell.

Practical solar cell structures cool down through nonradiative cooling. The top surface of the cell structure may be exposed to wind, while additional cooling systems may be put at the bottom of the cell. These nonradiative cooling mechanisms are characterized by the $h_{1}$ and $h_{2}$ coefficients in Eqs. (ㅁ) and (6). Here we evaluate the impact of radiative cooling as we vary the strength of these nonradiative cooling mechanisms. As an example, we fix the solar heating power to be $800 \mathrm{~W} / \mathrm{m}^{2}$. In general, as expected, as we increase the strength of nonradiative cooling mechanisms, the solar cell temperature decreases. The impact of radiative cooling, as measured by the temperature difference between the bare solar cell and the cell structures with radiative cooling layers, also decreases. Nevertheless, even in the presence of significant nonradiative cooling, radiative cooling can still have a significant impact. For example, as shown in Fig. 5(a), with $h_{1}=40 \mathrm{~W} / \mathrm{m}^{2} / \mathrm{K}$, which corresponds to a wind speed of $12 \mathrm{~m} / \mathrm{s}$ on the top surface [25], the temperature difference between the bare solar cell and the cell with silica pyramid is still as high as $5.3 \mathrm{~K}$. We also note that, in the presence of strong nonradiative cooling, the impact of radiative cooling is more significant in the thin silica pyramid structure as compared to the thicker uniform silica structure. This is related to 
(a)

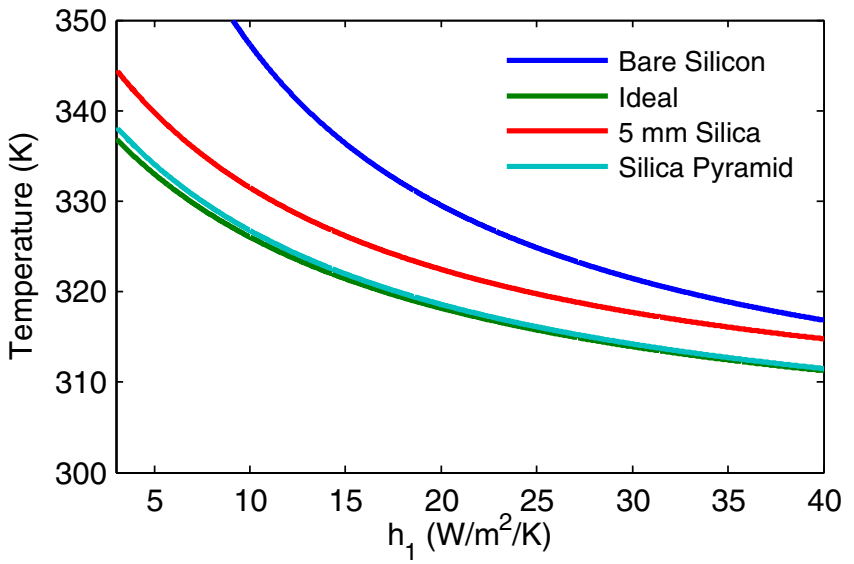

(b)

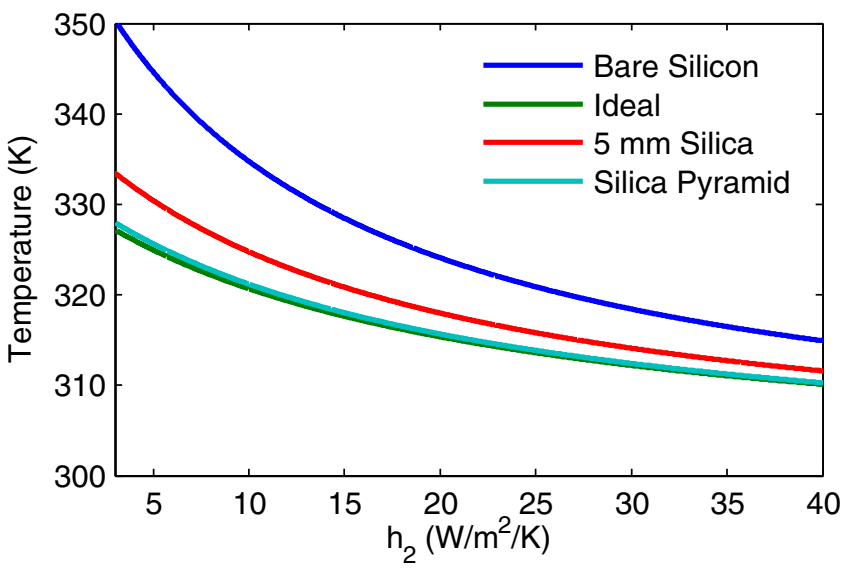

Fig. 5. (a) Operating temperature of the solar cell under different emitter designs, for different $h_{1}$, and fixed $h_{2}=6 \mathrm{~W} / \mathrm{m}^{2} / \mathrm{K}$. (b) Operating temperature of the solar cell under different emitter designs, for different $h_{2}$, and fixed $h_{1}=12 \mathrm{~W} / \mathrm{m}^{2} / \mathrm{K}$. The ambient temperature at both sides of the solar cell is $300 \mathrm{~K}$. The solar heating power is $800 \mathrm{~W} / \mathrm{m}^{2}$.

the larger thermal resistance in the thicker silica structure, which further diminishes the benefits of the radiative cooling.

In the simulations above, we have assumed the use of silica that is transparent in the solar wavelength range. In practice, the glass used as solar panel cover contains $70 \%-80 \%$ silica, with the rest being $\mathrm{Na}_{2} \mathrm{O}, \mathrm{CaO}, \mathrm{MgO}, \mathrm{Al}_{2} \mathrm{O}_{3}, \mathrm{~B}_{2} \mathrm{O}_{3}, \mathrm{~K}_{2} \mathrm{O}$, and $\mathrm{Fe}_{2} \mathrm{O}_{3}$ [37]. Glass, therefore, has a non-negligible amount of absorption in the solar wavelength range. To assess the sensitivity of the radiative cooling performance to absorption of solar irradiance inside the thermal emitter, we add a constant absorbance to the dielectric function of silica at solar wavelengths, for the devices in Figs. 1(c) and 1(d), and compute the resulting solar cell temperature as a function of the absorbance in the silica region, as shown in Fig. $\underline{6}$. In Fig. $\underline{6}$, the 5 -mm-thick uniform silica design is sensitive to possible absorption of solar irradiance inside the thermal emitter. With a relatively small absorbance of $0.2 \mathrm{~cm}^{-1}$, its operating temperature increases by $5.2 \mathrm{~K}$, reducing by nearly half the radiative cooling benefit of using 5 -mm-thick silica. In contrast, the performance of the silica pyramid design remains unchanged for this level of absorbance of solar irradiance inside the thermal

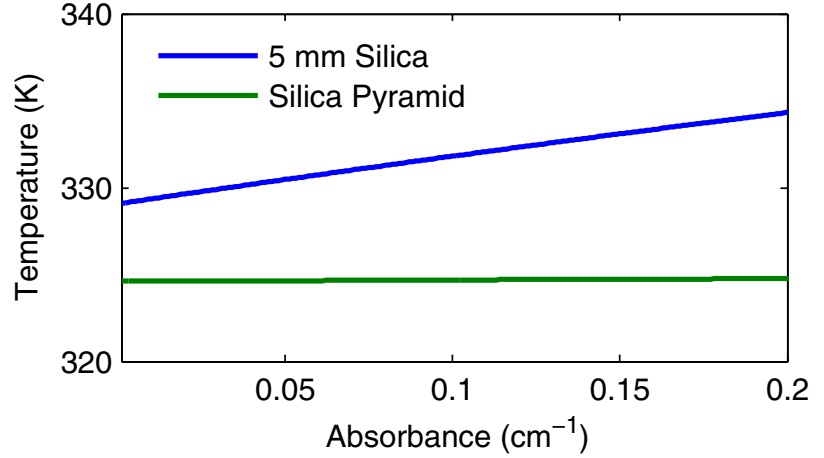

Fig. 6. Solar cell operating temperature, with a 5-mm-thick uniform silica layer (blue curve) and with the silica pyramid structure (green curve), where a constant absorbance at solar wavelengths has been artificially added to the material silica. $h_{1}=12 \mathrm{~m} / \mathrm{s}, h_{2}=6 \mathrm{~m} / \mathrm{s}$. The ambient temperatures at both sides of the solar cell is $300 \mathrm{~K}$. The solar heating power is $800 \mathrm{~W} / \mathrm{m}^{2}$.

emitter. The large contrast in the sensitivities to solar absorption inside the thermal emitter between the two designs results from the contrast in the thickness of the thermal emitter.

In summary, we have introduced the principle of radiative cooling of solar cells. We identify the ideal scheme as placing a thin, visibly transparent ideal thermal emitter atop the solar cell. While conventional solar cells have a thick cover glass panel, we show that such a glass panel can have only limited cooling performance due to its inherent thermal resistance and solar absorption. We have designed a thin, microphotonic thermal emitter based on silica pyramid arrays that approaches the performance of the ideal thermal emitter.

We remark on a few practical aspects related to our proposal. First of all, the choice of a crystalline silicon solar cell is not intrinsic to the performance of the radiative cooling, and the idea of utilizing microphotonic design to enhance thermal emission for solar cell radiative cooling should also apply to other types of solar cells. Second, in terms of experimental fabrication, nanocone or microcone structures with aspect ratio similar to our proposed pyramid structure here can be fabricated using various methods, including LangmuirBlodgett assembly and etching [38,39] and metal-dotted pattern and etching [40]. Therefore, our proposed pyramid structure should be within the regime where fabrication can be conducted. Third, it has been demonstrated in solar cells that a microstructure patterning [41] with aspect ratio similar to the silica pyramid, or nanostructure patterning [42], has superhydrophobicity and self-cleaning functionality. This functionality prevents dust accumulation, which would otherwise block sunlight and impair solar cell performance. Furthermore, patterning of microscale pyramids with rounded tips [ $\underline{43}$ ], or microcone patterning [44], has been shown to have superhydrophobicity and self-cleaning properties. Therefore, our proposed silica pyramid structure may readily have self-cleaning functionality, which prevents dust accumulation on solar cells, after a surface hydrophobilization process. As the surface hydrophobilization process only involves bonding a single-layer of hydrophobic molecules, it maintains optical transparency. Finally, the strict periodicity of the silica pyramid 
structure may not be necessary, as long as the structure possesses a spatial gradient in effective dielectric function to overcome the impedance mismatch between silica and air at thermal wavelengths.

Our study exploits an untapped degree of freedom for improving solar cell efficiency by engineering the thermal emission of solar cells through microphotonic design. Our analysis is based on direct simulation of $3 \mathrm{D}$ structures with realistic material properties, representing typical terrestrial photovoltaic operating conditions. The photonic thermal emitter design that approaches the maximal radiative cooling capability for solar cells may also provide additional opportunity for improving solar cell performance in space applications, where thermal radiation is the only cooling mechanism.

\section{FUNDING INFORMATION}

Advanced Research Projects Agency-Energy, U.S. Department of Energy (ARPA-E) (DE-AR0000316).

See Supplement 1 for supporting content.

\section{REFERENCES}

1. W. Shockley and H. J. Queisser, "Detailed balance limit of efficiency of p-n junction solar cells," J. Appl. Phys. 32, 510-519 (1961).

2. R. Santbergen and R. van Zolingen, "The absorption factor of crystalline silicon PV cells: a numerical and experimental study," Sol. Energy Mater. Sol. Cells 92, 432-444 (2008).

3. R. Santbergen, J. Goud, M. Zeman, J. van Roosmalen, and R. van Zolingen, "The AM1.5 absorption factor of thin-film solar cells," Sol. Energy Mater. Sol. Cells 94, 715-723 (2010).

4. J. Ingersoll, "Simplified calculation of solar cell temperatures in terrestrial photovoltaic arrays," J. Sol. Energy Eng. 108, 95-101 (1986).

5. A. Jones and C. Underwood, "A thermal model for photovoltaic systems," Sol. Energy 70, 349-359 (2001).

6. M. W. Davis, A. H. Fanney, and B. P. Dougherty, "Prediction of building integrated photovoltaic cell temperatures," J. Sol. Energy Eng. 123, 200-210 (2001).

7. E. Skoplaki and J. Palyvos, "On the temperature dependence of photovoltaic module electrical performance: a review of efficiency/power correlations," Sol. Energy 83, 614-624 (2009).

8. D. Otth and R. E. Ross, Jr., "Assessing photovoltaic module degradation and lifetime from long term environmental tests," Proceedings of the 1983 Institute of Environmental Sciences 29th Annual Meeting, Los Angeles, CA, April 19-21 1983, pp. 121-126.

9. A. Luque and G. Araújo, Solar Cells and Optics for Photovoltaic Concentration, Adam Hilger Series on Optics and Optoelectronics (Institute of Physics Publishing, 1989).

10. H. Teo, P. Lee, and M. Hawlader, "An active cooling system for photovoltaic modules," Appl. Energy 90, 309-315 (2012).

11. G. Mittelman, A. Kribus, and A. Dayan, "Solar cooling with concentrating photovoltaic/thermal (CPVT) systems," Energy Convers. Manage. 48, 2481-2490 (2007).

12. A. Akbarzadeh and T. Wadowski, "Heat pipe-based cooling systems for photovoltaic cells under concentrated solar radiation," Appl. Therm. Eng. 16, 81-87 (1996).

13. A. Royne, C. Dey, and D. Mills, "Cooling of photovoltaic cells under concentrated illumination: a critical review," Sol. Energy Mater. Sol. Cells 86, 451-483 (2005).

14. F. Trombe, "Perspectives sur l'utilisation des rayonnements solaires et terrestres dans certaines régions du monde," Rev. Gen. Therm. 6, 1285-1314 (1967).
15. S. Catalanotti, V. Cuomo, G. Piro, D. Ruggi, V. Silvestrini, and G. Troise, "The radiative cooling of selective surfaces," Sol. Energy 17, 83-89 (1975).

16. B. Bartoli, S. Catalanotti, B. Coluzzi, V. Cuomo, V. Silvestrini, and G. Troise, "Nocturnal and diurnal performances of selective radiators," Appl. Energy 3, 267-286 (1977).

17. A. Harrison and M. Walton, "Radiative cooling of $\mathrm{TiO}_{2}$ white paint," Sol. Energy 20, 185-188 (1978).

18. C. G. Granqvist and A. Hjortsberg, "Letter to the editor," Sol. Energy 24, 216 (1980).

19. C. G. Granqvist, "Radiative cooling to low temperatures: general considerations and application to selectively emitting SiO films," J. Appl. Phys. 52, 4205-4220 (1981).

20. P. Berdahl, "Radiative cooling with $\mathrm{MgO}$ and/or LiF layers," Appl. Opt. 23, 370-372 (1984).

21. G. Smith, "Amplified radiative cooling via optimised combinations of aperture geometry and spectral emittance profiles of surfaces and the atmosphere," Sol. Energy Mater. Sol. Cells 93, 1696-1701 (2009).

22. A. R. Gentle and G. B. Smith, "Radiative heat pumping from the Earth using surface phonon resonant nanoparticles," Nano Lett. 10, 373-379 (2010).

23. A. Gentle, J. Aguilar, and G. Smith, "Optimized cool roofs: integrating albedo and thermal emittance with R-value," Sol. Energy Mater. Sol. Cells 95, 3207-3215 (2011).

24. T. M. Nilsson, G. A. Niklasson, and C. G. Granqvist, "A solar reflecting material for radiative cooling applications: ZnS pigmented polyethylene," Sol. Energy Mater. Sol. Cells 28, 175-193 (1992).

25. T. M. Nilsson and G. A. Niklasson, "Radiative cooling during the day: simulations and experiments on pigmented polyethylene cover foils," Sol. Energy Mater. Sol. Cells 37, 93-118 (1995).

26. E. Rephaeli, A. Raman, and S. Fan, "Ultrabroadband photonic structures to achieve high-performance daytime radiative cooling," Nano Lett. 13, 1457-1461 (2013)

27. L. Zhu, A. Raman, and S. Fan, "Color-preserving daytime radiative cooling," Appl. Phys. Lett. 103, 223902 (2013).

28. T. Saga, "Advances in crystalline silicon solar cell technology for industrial mass production," NPG Asia Mater. 2, 96-102 (2010).

29. A. Goetzberger, J. Knobloch, and B. Voss, "High efficiency solar cells," in Crystalline Silicon Solar Cells (Wiley, 1998), Chap. 6, p. 122.

30. C. Fu and Z. Zhang, "Nanoscale radiation heat transfer for silicon at different doping levels," Int. J. Heat Mass Transfer 49, 1703-1718 (2006).

31. V. Liu and S. Fan, "S4: A free electromagnetic solver for layered periodic structures," Comput. Phys. Commun. 183, 2233-2244 (2012).

32. S. D. Lord, "A new software tool for computing Earth's atmospheric transmission of near- and far-infrared radiation," NASA Technical Memorandum 103957 (1992).

33. Gemini Observatory, IR Transmission Spectra, http://www.gemini .edu/?q=node/10789.

34. W. Haynes, CRC Handbook of Chemistry and Physics, 94th ed. (Taylor \& Francis, 2013)

35. United States Department of Energy, United States ResidentialScale 30-Meter Wind Maps, http://apps2.eere.energy.gov/wind/ windexchange/windmaps/residential_scale.asp.

36. E. Peterson and J. P. Hennessey, Jr., "On the use of power laws for estimates of wind power potential," J. Appl. Meteorol. 17, 390-394 (1978).

37. C. R. Kurkjian and W. R. Prindle, "Perspectives on the history of glass composition," J. Am. Ceram. Soc. 81, 795-813 (1998).

38. C.-M. Hsu, S. T. Connor, M. X. Tang, and Y. Cui, "Wafer-scale silicon nanopillars and nanocones by Langmuir-Blodgett assembly and etching," Appl. Phys. Lett. 93, 133109 (2008).

39. S. Wang, B. D. Weil, Y. Li, K. X. Wang, E. Garnett, S. Fan, and Y. Cui, "Large-area free-standing ultrathin single-crystal silicon as processable materials," Nano Lett. 13, 4393-4398 (2013).

40. H. Toyota and K. Takahara, "Fabrication of microcone array for antireflection structured surface using metal dotted pattern," Jpn. J. Appl. Phys. 40, L747-L749 (2001). 
41. Y.-B. Park, H. Im, M. Im, and Y.-K. Choi, "Self-cleaning effect of highly water-repellent microshell structures for solar cell applications," J. Mater. Chem. 21, 633 (2011).

42. J. Zhu, C.-M. Hsu, Z. Yu, S. Fan, and Y. Cui, "Nanodome solar cells with efficient light management and self-cleaning," Nano Lett. 10, 1979-1984 (2010).
43. M. Nosonovsky and B. Bhushan, "Roughness-induced superhydrophobicity: a way to design non-adhesive surfaces," J. Phys. Condens. Matter 20, 225009 (2008).

44. E. Hosono, S. Fujihara, I. Honma, and H. Zhou, "Superhydrophobic perpendicular nanopin film by the bottom-up process," J. Am. Chem. Soc. 127, 13458-13459 (2005). 